\title{
Dislocation nucleation during nanoindentation in a body-centered cubic TiZrHfNb high- entropy alloy
}

\author{
Y.X. Ye ${ }^{1}$, Z.P. Lu$^{2}$, T.G. Nieh ${ }^{1 *}$ \\ ${ }^{1}$ Department of Materials Science and Engineering, University of Tennessee, Knoxville, TN \\ 37996, USA \\ ${ }^{2}$ State Key Laboratory of Advanced Metals and Materials, University of Science and \\ Technology Beijing, Beijing 100083, China \\ * Corresponding author, e-mail: tnieh@utk.edu
}

\begin{abstract}
Pop-in behavior of a single-phase, body-centered cubic TiZrHfNb high-entropy alloy was characterized using instrumented nanoindentation. The critical shear stress required for the first pop-in was close to the theoretical strength, indicating it was controlled by dislocation nucleation. Data were collected and analyzed using a model based upon the transition-state theory and Weibull statistics. The activation volume for the pop-in events was evaluated to be about 3-5 atomic volumes, much larger than that in pure metals ( one atomic volume), suggesting cooperative migration of multiple atoms. The activation energy was also estimated and compared favorably with the nucleation of a full-dislocation.
\end{abstract}

Keywords: High-entropy alloy; Nanoindentation; Dislocation nucleation; Activation volume and energy

High-entropy alloys (HEAs) have attracted significant research attention since they were first introduced [1,2], because they have opened up a vast compositional space for alloy design. This new class of alloys has shown exceptional mechanical properties, such as good balance of strength-ductility property, excellent wear and fracture resistance [3-8]. Unlike conventional alloys with a major element, HEAs (also known as multi-principle element alloys) comprise of multiple equiatomic or nearly equiatomic metallic elements. The high configurational entropy over the mixing enthalpy promotes HEAs to form a simple solution-like phase, usually facecentered cubic (FCC) [8-10] or body-centered cubic (BCC) [11-13], instead of brittle intermetallics. This has been shown before [9] that, because the lack of a major diffusing element,

Page 1 of 9

(C) 2016. This manuscript version is made available under the Elsevier user license http://www.elsevier.com/open-access/userlicense/1.0/ 
atomic (or vacancy) migration in HEAs is expected to involve cooperative motion of several atoms in order to maintain proper composition portioning.

Instrumented nanoindentation has been demonstrated previously to be able to quantitatively assess the onset of yielding in crystals [9, 14-18]. Experimentally, plastic yielding is associated with distinct displacement bursts, or pop-ins, in the load-displacement curve. The first pop-in is often attributed to dislocation nucleation triggered by the applied shear stress near the theoretical strength $(\sim G / 10$, where $G$ is shear modulus) [9, 14-18]. Schuh et al. [14, 15] first proposed a statistical approach to quantitatively evaluate the temperature and rate dependence on the dislocation nucleation and extract related activation energy and volume in the single-crystal FCC-Pt. Recently, Zhu et al. [9] studied the nanoindentation pop-in behavior of a single-phase FCC-FeCoNiCrMn HEA and found the activation volume for dislocation nucleation is much larger than that in the conventional metals. It was also reported [18] that the pop-in behavior was quite different between FCC-Ni and BCC-Mo, indicating the effect of structure on dislocation nucleation. It is, therefore, of interest to investigate the atomic processes for dislocation nucleation in BCC-HEAs. In the present study, we perform instrumented nanoindentation to explore dislocation nucleation process in a BCC-TiZrHfNb HEA to compare it with that in conventional metals as well as FCC HEAs.

A high-entropy alloy with a nominal atomic composition of $\mathrm{Ti}_{25} \mathrm{Zr}_{25} \mathrm{Hf}_{25} \mathrm{Nb}_{25}$ was prepared by arc-melting a mixture of the constituent elements (purity > $99.9 \mathrm{wt} . \%$ ) in an argon atmosphere purified by a Ti-getter. The ingots were re-melted at least four times in high purity argon atmosphere to ensure the chemical homogeneity before being drop-cast into a water-cooled copper mold. The as-cast ingots were homogenized at $1290^{\circ} \mathrm{C}$ for $24 \mathrm{~h}$ and, then, cold rolled $80 \%$ in thickness. The rolled plates were additionally annealed at $1200^{\circ} \mathrm{C}$ for 30 mins to induce recrystallization and grain growth. Microstructure of the annealed samples has a grain size about $150 \mu \mathrm{m}$ examined by optical microscope and electron backscatter diffraction (EBSD, Fig. 1a). Phases and lattice parameter were identified by $\mathrm{X}$-ray diffraction (XRD) with $\mathrm{Cu}-\mathrm{K} \alpha$ radiation. Instrumented nanoindentation experiments were performed using a Berkovich diamond tip with an effective tip radius of $272 \mathrm{~nm}$ at room temperature $\left(22^{\circ} \mathrm{C}\right)$ on a Hysitron Triboindenter (Hysitron, Inc., Minneapolis, MN). To prevent possible overlap of the deformed zone produced by adjacent indentations, tests were conducted at a $5-\mu \mathrm{m}$ interval. Four randomly selected grains were tested at a fixed loading rate of $10 \mu \mathrm{N} / \mathrm{s}$. Various loading rates (10, 100 and $1000 \mu \mathrm{N} / \mathrm{s}$ ) 
were also carried out to investigate the rate dependence of the pop-in behavior. Testing samples for nanoindentation were prepared by initial mechanical polishing, followed by electropolishing to remove surface residual stresses. At least 120 nominally identical indentations were performed under each test condition to permit subsequent statistical analysis.

X-ray diffraction patterns obtained from the current TiZrHfNb HEA are shown in Fig.1b, in which diffraction peaks demonstrate a single-phase BCC structure. The lattice parameter was determined to be $3.444 \AA$. The lattice parameter can also be theoretically estimated from using the rule of mixture (i.e., Vegard's law [19]), $a_{\text {mix }}=\sum c_{i} a_{i}$, where $c_{i}$ and $a_{i}$ are atomic fraction and lattice parameter of the alloying element $i$, respectively. It is pointed out that $\mathrm{Ti}, \mathrm{Zr}$ and $\mathrm{Hf}$ have a hexagonal close-packed (HCP) structure at room temperature, but transform to BCC structure at high temperatures. Thus, lattice parameters of $\mathrm{BCC}-\mathrm{Ti}, \mathrm{Zr}$ and $\mathrm{Hf}$ at room temperature were estimated from the extrapolated values by using thermal expansion data measured at high temperatures [20]. With these lattice parameters of constituent metals ( $a_{T i}$ $=3.276 \AA, a_{Z r}=3.582 \AA, a_{H f}=3.559 \AA$ and $\left.a_{N b}=3.301 \AA\right), a_{m i x}(3.430 \AA)$ appears to agree well with the experimental value (3.444 $\AA$ ), indicating the rule-of-mixture calculation is actually a reasonable approximation in this particular HEA.

Typical load-displacement $(P-h)$ curve is shown in Fig. 2a, which exhibits several discrete bursts of displacement, termed as "pop-ins", of which the first one is normally regarded as the onset of plasticity. Prior to the first pop-in, the initial elastic portion of the load-displacement curve is well described (red curve in Fig. 2a) by the Hertzian elastic contact theory [21]: $P=(4 / 3) E_{r} \sqrt{R h^{3}}$, where $P$ is the applied indenting load, $R$ is the tip radius of indenter, $h$ is the indentation depth, and $E_{r}=\left[\left(1-v_{i}^{2}\right) / E_{i}+\left(1-v_{s}^{2}\right) / E_{s}\right]^{-1}$ is the reduced elastic modulus for a diamond indenter on HEA sample. In the reduced elastic modulus equation, $v_{i}=0.07$ and $E_{i}=1141$ $\mathrm{GPa}$ are the Poisson's ratio and Young's modulus of the diamond indenter, respectively, $v_{s}=0.26$ and $E_{s}$ are the Poisson's ratio and Young's modulus of the HEA specimen, respectively. Statistic distribution of more than 480 indentation $P-h^{3 / 2}$ pairs at pop-ins is given in Fig. 2b. From the linearly fit (red line in Fig. 2b), the reduced modulus and Young's modulus are readily deduced to be $83.0 \pm 0.6$ and $83.7 \pm 0.6 \mathrm{GPa}$, respectively. 
According to Hertz's contact theory [21], the maximum shear stress $\tau_{\max }$ beneath a spherical indenter occurs at a location of approximately 0.48 of the contact radius underneath the indenter tip with a value [15]

$$
\tau_{\max }=\left(\frac{0.47}{\pi}\right)\left(\frac{4 E_{r}}{3 R}\right)^{2 / 3} P^{1 / 3}
$$

Typical cumulative probability distribution against the maximum shear stress at the first pop-in, so-called nanoscale strength distribution (NSD), is shown in Fig. 2c. The maximum shear stress is noted to be in the range of 2.5-3.1 GPa, falling within the region of $G / 13-G / 10$, where $G$ is the shear modulus of TiZrHfNb-HEA ( $\sim 33 \mathrm{GPa}$, estimated from the Young's modulus). The proximity to the theoretical strength $(\sim G / 10)$ indicates the onset of plasticity is triggered by a dislocation nucleation mechanism. To investigate the grain orientation effect, the cumulative probability plot for pop-in events from 4 different grains is shown in the right-hand inset of Fig 2c. The corresponding crystal orientation of these grains for indentation was shown in the upper left inset. At least 120 identical indentations were performed on each grain. The overlap of NSD curves from different grains suggests insignificant crystallographic orientation dependence for pop-in events in the present BCC-HEA. This insensitive grain orientation dependence is similar to that observed in a FCC-HEA [9].

It is generally recognized that the first nanoindentation pop-in is caused by a dislocation nucleation process $[9,14,22-24]$. The fact that the maximum shear stress required for pop-ins in the present BCC-HEA approaches the theoretical strength also supports this hypothesis. To shed lights on the exact nucleation process, let us evaluate the activation volume employing a method proposed by Schuh and his co-workers [15, 16], which combines the transition-state theory [25] and Weibull-type statistics [26]. In the analysis, the dislocation nucleation is treated as a thermally activated, stress-assisted process, and the dislocation nucleation rate at any lattice site can be described through an Arrhenius equation $\dot{n}=\dot{n}_{0} \cdot \exp \left(-\Delta \prod / k T\right)$, where $\Delta \Pi$ is the stressbiased activation energy for dislocation nucleation $\left(\Delta \prod=\varepsilon-\tau v^{*}\right), \dot{n}_{0}$ the attempt frequency per unit volume, $\varepsilon$ the intrinsic nucleation energy barrier, $v^{*}$ the activation volume, $k$ the Boltzmann's constant and $T$ the absolute temperature. The local rate can be integrated over the indented volume underneath the indenter $(V)$ to deduce the global nucleation rate $(\dot{N})$ at which 
pop-in would occur, that is, $\dot{N}=\dot{n}_{o} \cdot \exp \left(-\frac{\varepsilon}{k T}\right) \cdot \iiint_{V} \exp \left(\tau v^{*} / k T\right) d V$, where $V$ scales with the cube of contact radius $V \approx \pi a^{3}=\pi\left(3 P R / 4 E_{r}\right)$. Link this nucleation rate equation to Weibull statistics, the final cumulative probability is $F(t)=1-\exp \left(-\int_{0}^{t} \dot{N}(t) d t\right)$. With the relationship between load (or stress $\tau_{\max }$, which scales with $P^{1 / 3}$ ) and time, the cumulative probability is eventually expressed as (see Ref. [15] for details)

$$
F\left(\tau_{\text {max }}\right)=1-\exp \left[-\frac{9 \pi R \eta}{4 E_{r} \dot{P} \alpha^{6}} \cdot \gamma\left(\tau_{\max }\right)\right]
$$

where $\dot{P}$ is the loading rate, $\eta=\dot{N}_{0} \cdot \exp (-\varepsilon / k T)$ is the nucleation rate without stress assistance, $\gamma\left(\tau_{\max }\right)$ is a complex function of $\tau_{\max }, \alpha=(0.47 / \pi)\left(4 E_{r} / 3 R\right)^{2 / 3}\left(v^{*} / k T\right)$ is a collection of timeindependent terms. The activation volume can be further obtained via the equation of $\ln [-\ln (1-F)]=\alpha P^{1 / 3}+\beta$, where $\beta$ is a much weaker function of the load as compared to the first term on the right-hand side [15]. Converting the load into stress in Eq. (1) and the parameter $\alpha$, we get

$$
\ln [-\ln (1-F)]=\frac{v^{*}}{k T} \tau_{\max }+\beta
$$

It is readily seen that the activation volume can be extracted from Eq. (3), thus a plot of $\ln [-\ln (1$ $F)$ ] versus $\tau$ is subsequently constructed, as shown in Fig. 2d. The activation volume, obtained from the slope of the fitted curve in the figure, is about $70 \AA^{3}$, which corresponds to $1.7 a_{0}^{3}$, where $a_{0}$ is the lattice parameter of the alloy, or about $3 \Omega$, where $\Omega$ is the atomic volume. The parameter $\beta$, associated with $\eta$, can be also obtained from the intercept of the solid red lines with $\tau=0$ in Fig. 2d. Once we have all the parameters in Eq. (2), the predicted cumulative probability for the first pop-ins can be drawn as the solid red line in Fig. 2c. Despite of slight deviations at the two ends of the Heaviside-like curve, which may be attributed to the tip radius effect [27], Eq. (2) is quite accurate in describing the pop-in behavior.

There is yet another method to evaluate the activation volume, which involves the measurement of the strain rate sensitivity value, $m$. To obtain the $m$ value, we conducted tests at different indentation rates. The effect of rate dependence on the onset of plasticity is shown in Fig. 3a. It is evident that the maximum shear stress required for the pop-in event increases with 
increasing loading rate in the present TiZrHfNb-HEA. This is consistent with the notion that, during a stress-assisted thermally activated nucleation process [14-16], an increased time-at-load would result in a higher probability for a critical thermal fluctuation to occur. This phenomenon has been also observed in many metals, such as BCC-Ta [23], FCC-Pt [14, 15] and HCP-Mg [17]. The strain rate dependence of the present BCC-HEA, however, appears to be less prominent than that in the single-crystal FCC-Pt $[14,15]$.

The mean maximum shear stress versus strain rate plotted in a double logarithmic scale is given in Fig. 3b, where the strain rate sensitivity $m$ is evaluated as 0.014. For a thermally activated process, the activation volume can be simply calculated using the equation $v^{*}=k T / \tau m$ [28]. Insert the current $m$ value, the activation volume is computed as $2.4 a_{0}{ }^{3}$, corresponding to $\sim 5 \Omega$, which is slightly larger than that estimated using the former statistical method. The discrepancy by using the two methods is acceptable considering the fact that the rate data used in Fig. $3 \mathrm{~b}$ is the loading rate not strain rate, even though they often linearly scale with each other [29, 30]. Furthermore, data variation in delicate nanoindentation measurements is always expected. Nevertheless, both methods yield a similar magnitude of $v^{*}$ for the pop-in events and, specifically, it is greater than $\Omega$.

Activation volume is a good indicator to reveal the atomic process for dislocation nucleation. Activation volume data for some BCC and FCC materials [9, 14, 18] are summarized on Table 1. It is noted that the activation volume for the conventional FCC and BCC metals is in the order of $\Omega$, suggesting the migration of individual point-like defect (e.g., vacancy or impurity). In contrast, the activation volumes for both FCC-HEA [9] and the present BCC-HEA are approximately 3-5 $\Omega$, indicating that dislocation nucleation in HEAs is relatively difficult, involving cooperative migration of several atoms.

In addition to having a slightly higher activation volume in BCC-HEA as compared to FCCHEA, it is of interest to compare the activation energy for dislocation nucleation between the two types of HEAs. Accumulative probability curves, thus the pop-in behavior, of the current BCCHEA and previously studied FCC-HEA are directly compared in Fig. 4. In the figure, the shear stress in the $\mathrm{x}$-axis is presented in a normalized form, since these two materials have significantly different values of shear modulus. It is apparent that the curve for the BCC-HEA is much steeper as compared to that for the FCC-HEA. According to continuum dislocation analysis [31, 32], the activation energy $U$ for dislocation nucleation can be calculated via the following equation 
$\Delta U=A\left(1-\tau_{\text {app }} / \tau_{t h}\right)^{n}[33,34]$, where $\tau_{\text {app }}$ is the applied shear stress, $\tau_{\text {th }}$ is the theoretical stress corresponding to the upper bound of the maximum shear stress, the exponent $n$ (in the range of $1.5-4.5$ ) and the pre-factor $A$ (in the range of (5-15) $G b^{3}$ with $b$ being the Burgers vector) are fitting parameters [33-35]. For a first-order approximation, the cumulative probability for pop-in is given by, $F=1-q=1-\exp \left[-\dot{n}_{0} \cdot V \cdot \int_{0}^{p_{\text {pop-in }}} \exp (-\Delta U / k T) d P / \dot{P}\right][18,34,35]$, where $V$ in the equation is the indented volume and $q$ is the survivability (i.e., the probability that no pop-in occurs), or

$$
F=1-\exp \left[-\dot{n}_{0} \cdot V \cdot \frac{\tau_{t h} \cdot k T}{n A\left(1-\tau_{a p p} / \tau_{t h}\right)^{n-1} \cdot \dot{\tau}} \cdot \exp \left(-\frac{A\left(1-\tau_{a p p} / \tau_{t h}\right)^{n}}{k T}\right)\right]
$$

This equation can nicely fit the experimental curves for the BCC-HEA, as shown in Fig. 4. In this case, the fitted cumulative probability yields reasonable values of $n=3.5, A \approx 11 G b^{3}$ with Burgers vector corresponding to the magnitude of $<111>/ 2$ full dislocation. However, in the case of FCC-HEA, only using $<11 \overline{2}>/ 6$ partial dislocation would yield reasonable values of $n=4.5$, $A \approx 6 G b^{3}$. Fitting the cumulative probability curve with Burgers vector $<110>/ 2$ would not yield reasonable values of $n$ and $A$ (specifically, $n=14$ and $A \approx 3 G b^{3}$ are both beyond the reasonable ranges [33-35]). This suggests that dislocation nucleation in BCC-HEA is a full dislocation, whereas a partial dislocation is favored in FCC-HEA. Consequently, a steeper $f-\tau / G$ curve for the BCC-HEA is a result of higher activation energy for full dislocation nucleation. In comparison, for the FCC-HEA, lower activation energy is required for partial dislocation nucleation. Similar observation has been reported before in conventional BCC-Mo and FCC-Ni metals [18].

In summary, we have conducted a series of instrumented nanoindentation tests to investigate dislocation nucleation in a single-phase BCC-TiZrHfNb HEA. The maximum shear stress required for dislocation nucleation in the alloy is about $1 / 10$ of the shear modulus. Differed from conventional metals, the activation volume for dislocation nucleation in the present HEA is estimated to be $\sim 3-5$ atomic volumes, indicative of a process of cooperative migration of multiple atoms. The activation energy for pop-ins in the current BCC-HEA is also found to be much higher than that in the typical FCC-HEA, which is attributable to the fact that the 
nucleation of full dislocation is favored in BCC-HEAs, whereas nucleation of partial dislocation is energetically favored in FCC-HEAs.

This work was supported by the National Science Foundation under Contract NSF DMR1408722. Instrumentation for the nanoindentation work was jointly funded by the Tennessee Agricultural Experiment Station and University of Tennessee College of Engineering. ZPL would like to acknowledge the financial support from National Natural Science Foundation of China (Nos. 51531001, 51671018, 51422101, 51271212 and 51371003), 111 Project (B07003), International S\&T Cooperation Program of China (2015DFG52600) and Program for Changjiang Scholars and Innovative Research Team in University (IRT_14R05).

[1] J.W. Yeh, S.K. Chen, S.J. Lin, J.Y. Gan, T.S. Chin, T.T. Shun, C.H. Tsau, S.Y. Chang, Adv. Eng. Mater. 6 (2004) 299-303.

[2] B. Cantor, I.T.H. Chang, P. Knight, A.J.B. Vincent, Mater. Sci. Eng. A 375 (2004) 213-218.

[3] Y. Zhang, T.T. Zuo, Z. Tang, M.C. Gao, K.A. Dahmen, P.K. Liaw, Z.P. Lu, Prog. Mater. Sci. 61 (2014) 1-93.

[4] M.H. Chuang, M.H. Tsai, W.R. Wang, S.J. Lin, J.W. Yeh, Acta Mater. 59 (2011) 6308-6317.

[5] J.Y. He, H. Wang, H.L. Huang, X.D. Xu, M.W. Chen, Y. Wu, X.J. Liu, T.G. Nieh, K. An, Z.P. Lu, Acta Mater. 102 (2016) 187-196.

[6] W.H. Liu, Z.P. Lu, J.Y. He, J.H. Luan, Z.J. Wang, B. Liu, Y. Liu, M.W. Chen, C.T. Liu, Acta Mater. 116 (2016) 332-342.

[7] Z. Li, K.G. Pradeep, Y. Deng, D. Raabe, C.C. Tasan, Nature 534 (2016) 227-230.

[8] B. Gludovatz, A. Hohenwarter, D. Catoor, E.H. Chang, E.P. George, R.O. Ritchie, Science 345 (2014) 1153-1158.

[9] C. Zhu, Z.P. Lu, T.G. Nieh, Acta Mater. 61 (2013) 2993-3001.

[10] Z. Fu, W. Chen, H. Wen, D. Zhang, Z. Chen, B. Zheng, Y. Zhou, E.J. Lavernia, Acta Mater. 107 (2016) 59-71.

[11] S. Maiti, W. Steurer, Acta Mater. 106 (2016) 87-97.

[12] N.N. Guo, L. Wang, L.S. Luo, X.Z. Li, R.R. Chen, Y.Q. Su, J.J. Guo, H.Z. Fu, Intermetallics 69 (2016) 13-20.

[13] O.N. Senkov, G.B. Wilks, J.M. Scott, D.B. Miracle, Intermetallics 19 (2011) 698-706. 
[14] C.A. Schuh, J.K. Mason, A.C. Lund, Nature Materials 4 (2005) 617-621.

[15] J.K. Mason, A.C. Lund, C.A. Schuh, Phys. Rev. B 73 (2006) 054102.

[16] C.A. Schuh, A.C. Lund, J. Mater. Res. 19 (2004) 2152-2158.

[17] H. Somekawa, C.A. Schuh, Acta Mater. 59 (2011) 7554-7563.

[18] L. Wang, H. Bei, T.L. Li, Y.F. Gao, E.P. George, T.G. Nieh, Scripta Mater. 65 (2011) 179182.

[19] L. Vegard, Z. Physik 5 (1921) 17-26.

[20] O.N. Senkov, J.M. Scott, S.V. Senkova, D.B. Miracle, C.F. Woodward, J. Alloys Compd. 509 (2011) 6043-6048.

[21] K.L. Johnson, Contact Mechanics, Cambridge University Press, Cambridge, 1987.

[22] H. Bei, Y.F. Gao, S. Shim, E.P. George, G.M. Pharr, Phys. Rev. B 77 (2008) 060103.

[23] M.M. Biener, J. Biener, A.M. Hodge, A.V. Hamza, Phys. Rev. B 76 (2007) 165422.

[24] D.F. Bahr, G. Vasquez, J. Mater. Res. 20 (2005) 1947-1951.

[25] P. Hänggi, P. Talkner, M. Borkovec, Rev. Mod. Phys. 62 (1990) 251.

[26] W. Weibull, J. Appl. Mech. 103 (1951) 293-297.

[27] D. Wu, J.R. Morris, T.G. Nieh, Scripta Mater. 94 (2015) 52-55.

[28] Q. Wei, S. Cheng, K.T. Ramesh, E. Ma, Mater. Sci. Eng. A 381 (2004) 71-79.

[29] C.A. Schuh, T.G. Nieh, Y. Kawamura, J. Mater. Res. 17 (2002) 1651-1654.

[30] C.L. Wang, M. Zhang, T.G. Nieh, J. Phys. D: Appl. Phys. 42 (2009) 115405.

[31] J.R. Rice, R. Thomson, Philos. Mag. 29 (1974) 73-97.

[32] J.P. Hirth, J. Lothe, Theory of Dislocations, second ed., John Wiley \& Sons, New York, 1982

[33] T. Zhu, J. Li, A. Samanta, A. Leach, K. Gall, Phys. Rev. Lett. 100 (2008) 025502.

[34] Y. Gao, H. Bei, Prog. Mater. Sci. 82 (2016) 118-150.

[35] T.L. Li, Y.F. Gao, H. Bei, E.P. George, J. Mech. Phys. Solids 59 (2011) 1147-1162. 
Table 1 Summary of activation volume for the pop-in in some BCC and FCC materials.

\begin{tabular}{cccccc}
\hline Materials & FCC-Pt [14] & FCC-Ni [18] & BCC-Mo [18] & FCC-HEA [9] & BCC-HEA \\
\hline $\begin{array}{c}\text { Activation } \\
\text { volume }(\Omega)\end{array}$ & $\sim 0.5$ & $\sim 1$ & $\sim 1$ & $\sim 3$ & $\sim 3-5$ \\
\hline
\end{tabular}





Fig. 1 (a) EBSD image and (b) X-ray diffraction patterns of the TiZrHfNb HEA. Indexed peaks indicate a single-phase BCC crystal structure with a lattice parameter of $3.444 \AA$. 

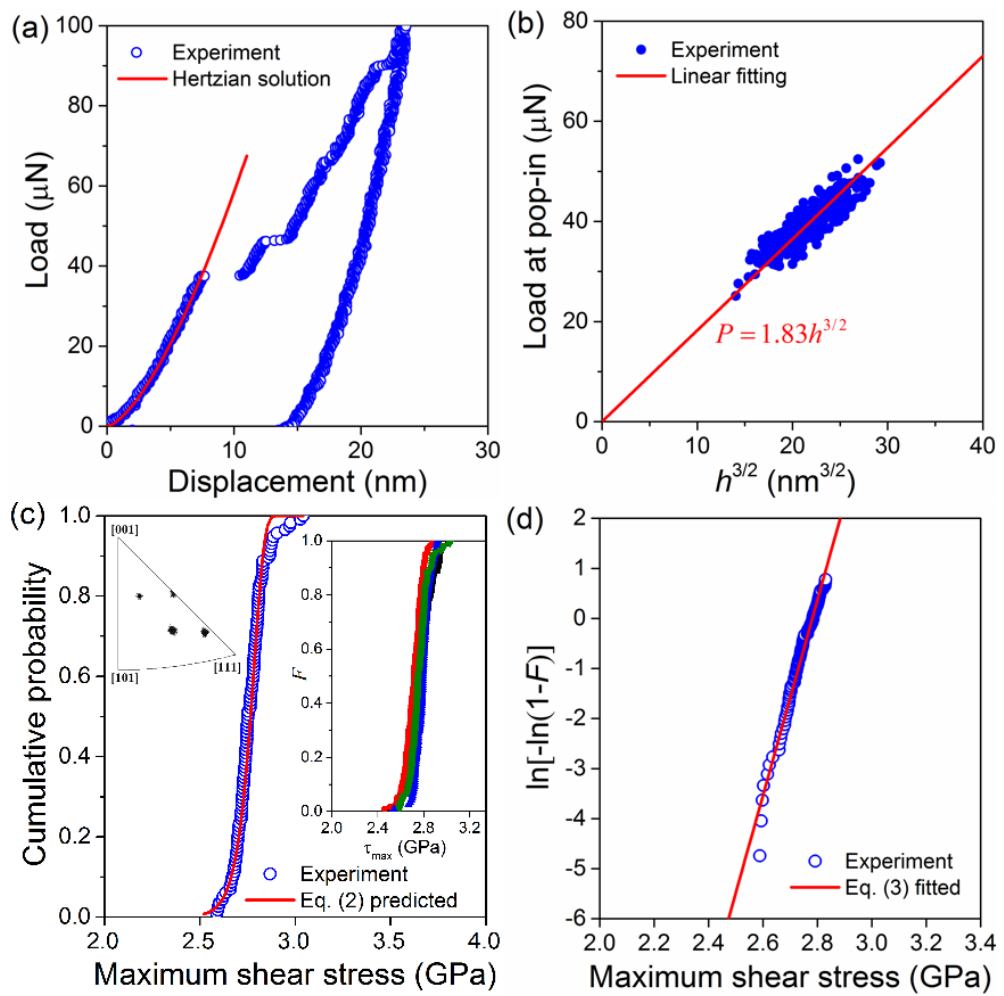

Fig. 2 (a) A representative load-displacement curve showing pop-in events; (b) statistics of 480 $P-h^{3 / 2}$ pairs at pop-ins; (c) cumulative probability of the first pop-in as a function of maximum shear stress, with the right-hand inset showing negligible crystallographic orientation dependence from 4 different grains and the upper left inset showing the corresponding crystal orientation; and (d) the relation between $\ln [-\ln (1-F)]$ and $\tau_{\max }$. 

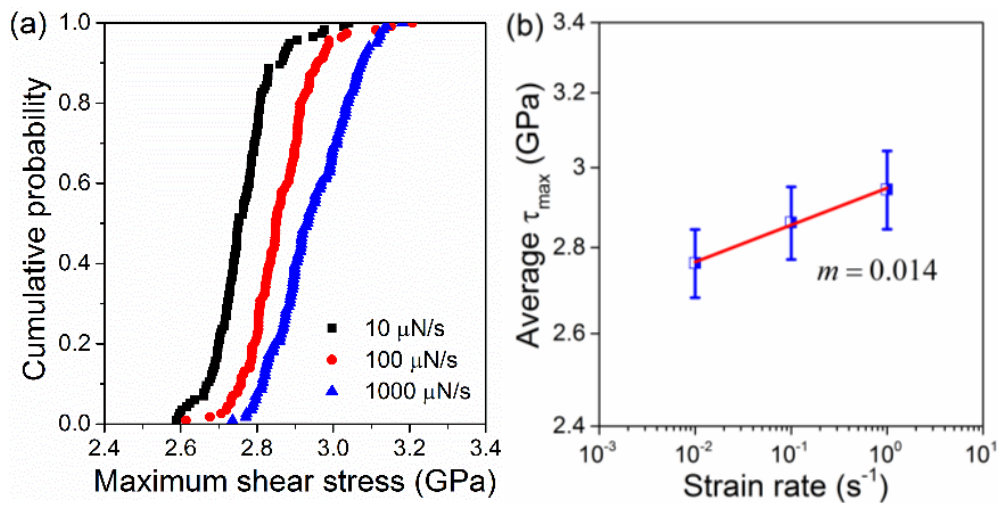

Fig. 3 (a) Plot of cumulative probability versus the maximum shear stress at various loading rates, and (b) double logarithmic plot of the average maximum shear stress as a function of the strain rate. 


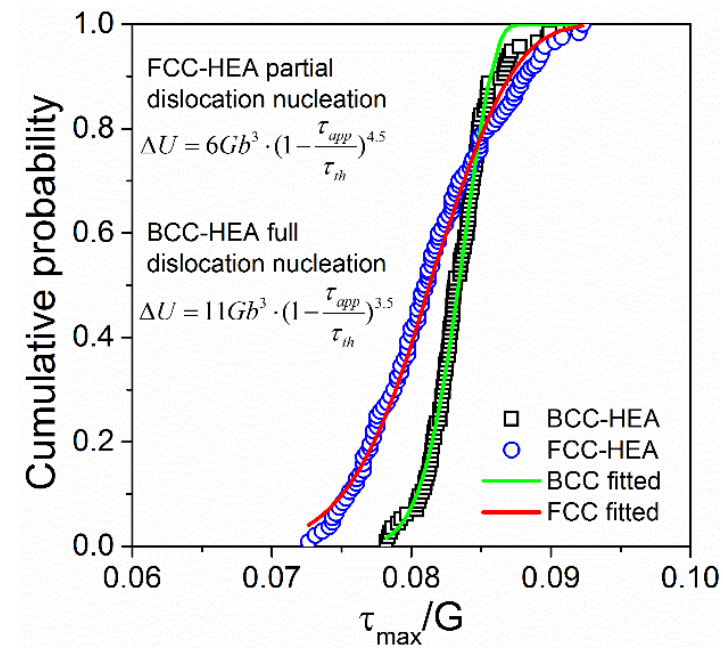

Fig. 4 Plot of cumulative probability of pop-in versus normalized shear stress for the current BCC-HEA and a FCC-HEA [9], and solid curves are fittings of Eq. (4). 

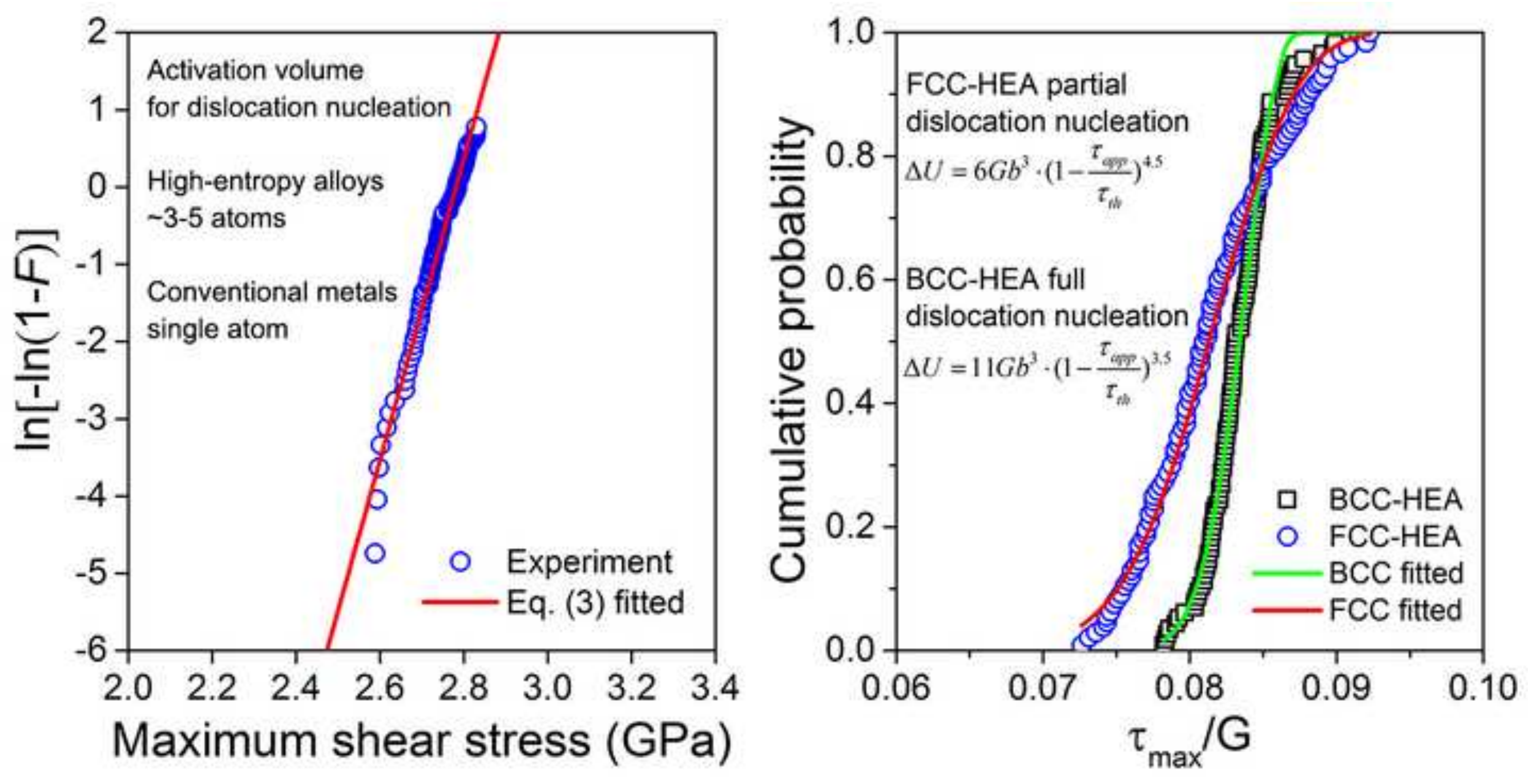